\title{
An In Silico Screening on Piper nigrum, Syzygium aromaticum and Zingiber officinale roscoe Derived Compounds Against SARS-CoV-2: A Drug Repurposing Approach
}

\author{
Pratibha Pandey 1,* (D), Divyanshi Singhal ${ }^{1}$, Fahad Khan ${ }^{1, *}$, Mohd Arif ${ }^{1}$ \\ 1 Department of Biotechnology, Noida Institute of Engineering and Technology, Greater Noida, India \\ * Correspondence: shukla.pratibha1985@gmail.com (P.P.); fahadintegralian@gmail.com (F.K.); \\ Scopus Author ID 54985258500 (P.P.); 57201049984 (F.K.) \\ Received: 9.11.2020; Revised: 4.12.2020; Accepted: 5.12.2020; Published: 9.12.2020
}

\begin{abstract}
As of now, Coronavirus (COVID-19) is spreading overall quickly, and its control is troublesome because there is no compelling immunization or medications accessible in the medical sector. This contagious disease has been associated with numerous respiratory issues. Thus, there is a crucial need to elucidate plant-derived compounds that display inhibitory potential against potential targets of coronavirus and boost the human body's immunity. This infection can contaminate the individuals and cause diseases of the respiratory lot. This research has focused on exploiting the medicinal properties of phytocompounds of three plants that have shown significant anti-inflammatory potential and had been effective against numerous respiratory disorders. This research's main objective was to study the inhibitory potential of these selected twenty-seven phytocompounds derived from Piper nigrum, Syzygium aromaticum, and Zingiber officinale roscoe against protease of COVID-19. We performed screening of selected phytocompounds with antivirus action by employing different in silico approaches, including Lipinski rule of five, adme analysis, and molecular docking tools. In silico investigation has revealed the inhibitory potential of these selected ligands (phytocompounds), two crucial targets of coronavirus, including 6LU7 and 7JTL. Out of 27 selected phytocompounds guaiol and gingeronone A has displayed significant inhibitory potential against coronavirus's selected targets. Thus our research findings strongly recommended that phytocompounds derived from black pepper, clove, and ginger could be very useful in battling the COVID-19 pandemic era.
\end{abstract}

Keywords: COVID-19; black pepper; clove; ginger; 7JTL; 6 LU7; molecular docking; drug repurposing.

(C) 2020 by the authors. This article is an open-access article distributed under the terms and conditions of the Creative Commons Attribution (CC BY) license (https://creativecommons.org/licenses/by/4.0/).

\section{Introduction}

COVID-19 contamination was first depicted in December 2019 in Wuhan, China. Since at that point, this ailment has dispersed through most nations worldwide and as of now caused in excess 9 lakhs fatalities [1]. The causative operator of COVID-19, for example, Serious Acute Respiratory Syndrome-Coronavirus-2 (SARS-CoV-2), is a novel beta-corona infection that imparts similitudes to SARS and Middle East Respiratory Syndrome (MERS) infections, which were beforehand answerable for endemics [2-5]. Current treatments are mainly focusing on helicase, protease, immunomodulators, and polymerase, including interferons and corticosteroids [6-9], even though RT-PCR remains the reference standard for making an authoritative finding of COVID-19 disease. Also, bioinformatics plays an important role in 
designing new drugs, which would effortlessly help decrease the hour of the investigation, probability of mistake, and the significant expense of clinical and research center preliminaries [10-12]. One of the novel helpful methodologies utilized for the restraint of infection disease is looking for an inhibitor of the protein in normal mixes as they have insignificant results. So, our study was focused on Black pepper (Piper nigrum), Clove(Syzygium aromaticum), and Ginger(Zingiber officinale roscoe). The physical and chemical properties of derived compounds have the capacity to impede the development of protein and hereditary material in the virus infection. Black pepper, clove, and ginger have anti-inflammatory, antibacterial, antiviral, and other healthful properties [13-18]. Continuing exploration, our current examination is intended to discover influential regular helpful specialists from black pepper, clove, and ginger that could show better inhibitory viability against the principle protease of COVID-19 by utilizing the molecular docking approach. This examination's aftereffects will furnish better chances to different specialists with better approaches to perceive and improve new COVID-19 treatment. Until any precise treatment philosophy is accessible for COVID19, the utilization of subsidiaries of recently realized antiviral medications is a helpful procedure. In this investigation, docking studies were performed over restricting the pocket of COVID-19 to locate the likely little particle to battle perilous COVID-19 illness.

\section{Materials and Methods}

\subsection{Tools requirements for in-silico analysis.}

Windows 7 or Windows 10, MGL tools, Discovery Studio Visualizer, Autodock 4.2, Cygwin, Binary files

\subsection{Preparation of receptor (target structure).}

The objective utilized for docking is the significant protease of the novel COVID-19 and SARS-CoV-2. Their 3D structures were downloaded from PDB (Protein Data Bank), having PDB ID: 6LU7 and 7JTL in pdb format (PDB url:-www.rcsb.org). During target preparation, water particles were eliminated.
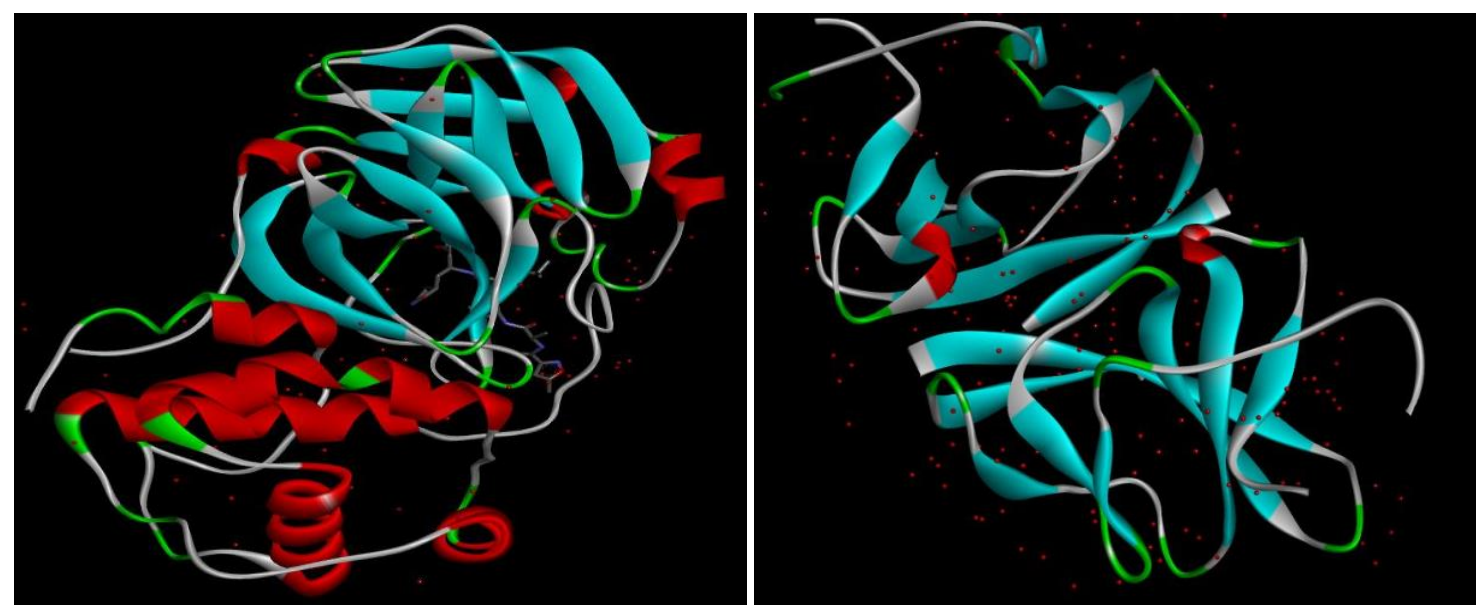

Figure 1. 3-dimensional structure of COVID-19 targets (A) 6LU7 and (B) 7JTL selected for docking analysis.

\subsection{Ligand selection and preparation.}

Compounds possessing antiviral activity from Black pepper (Piper nigrum), Clove (Syzygium aromaticum), and Ginger (Zingiber officinale roscoe) were selected for docking 
analysis. Their 3-Dimensional structures were obtained from PubChem in .sdf format (PubChem url:- https://pubchem.ncbi.nlm.nih.gov/).

\subsection{Lipinski's rule of five.}

All the compounds were evaluated for their oral bioavailability and medication likeliness properties by Lipinski's standard of five [19-20]. The natural mixes of black pepper, clove, and ginger are picked for docking analysis. The chemical structures of these compounds, along with their PubChem ID, are given in Table 1. These whole sets of preliminary screening probably affect the pharmacokinetics adequacy of medications [Table 2]. Further docking studies were executed according to the protocol described by Khan et al., 2019 [21].

Table 1. Compounds of various compounds from Black pepper (Piper nigrum), Clove (Syzygium aromaticum), and Ginger (Zingiber officinale roscoe).

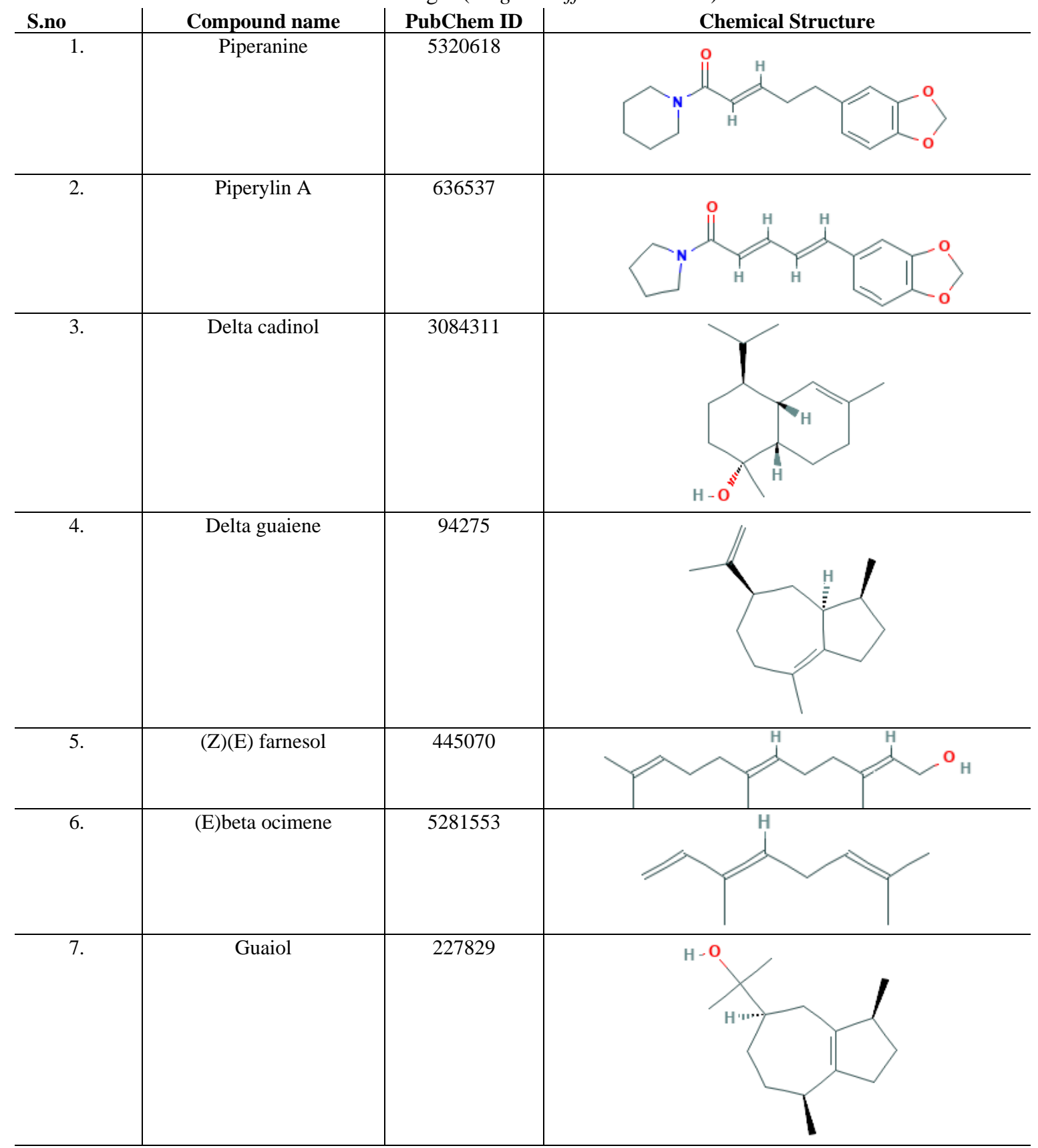

Phytocompounds of Syzygium aromaticum (clove) 


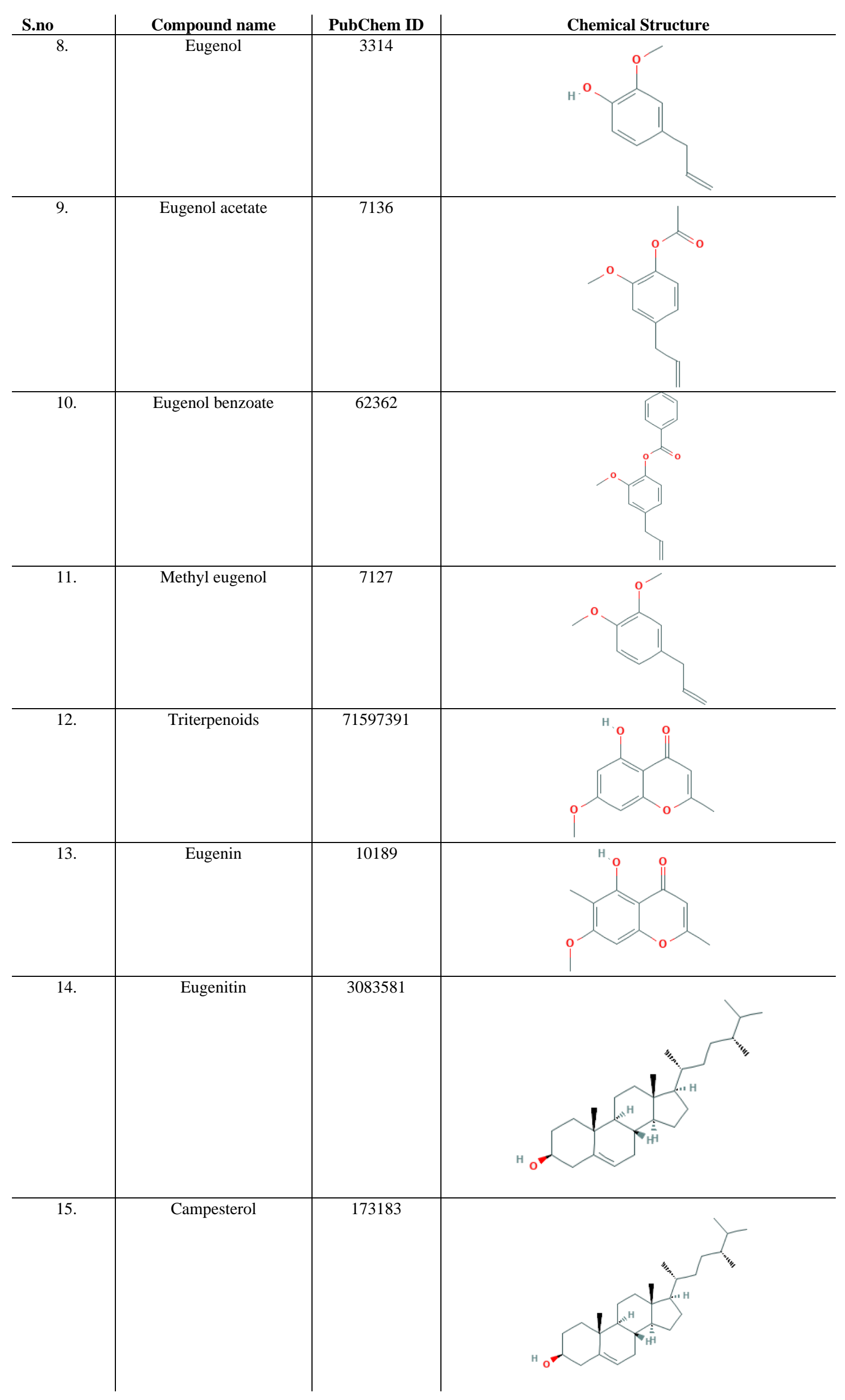




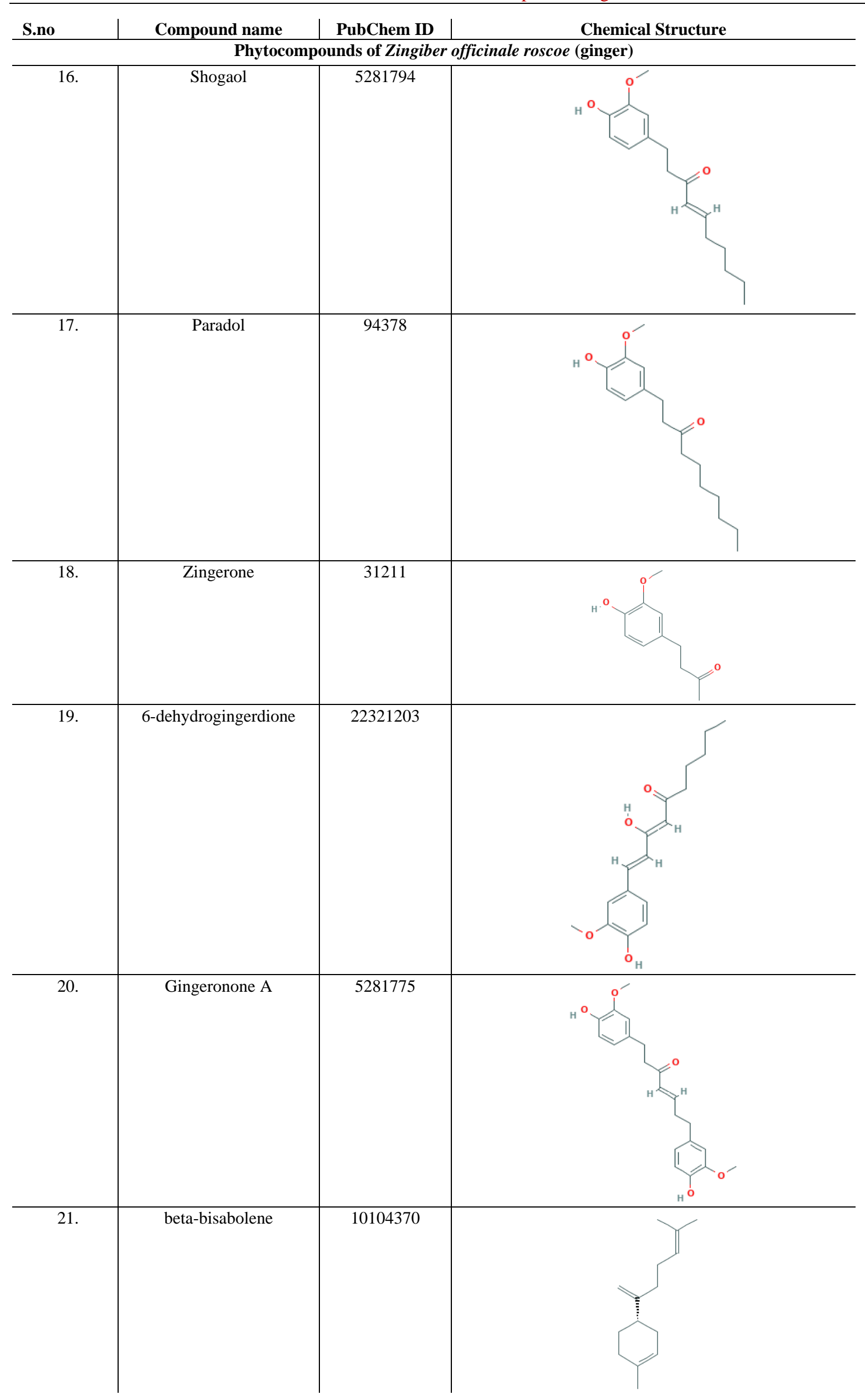




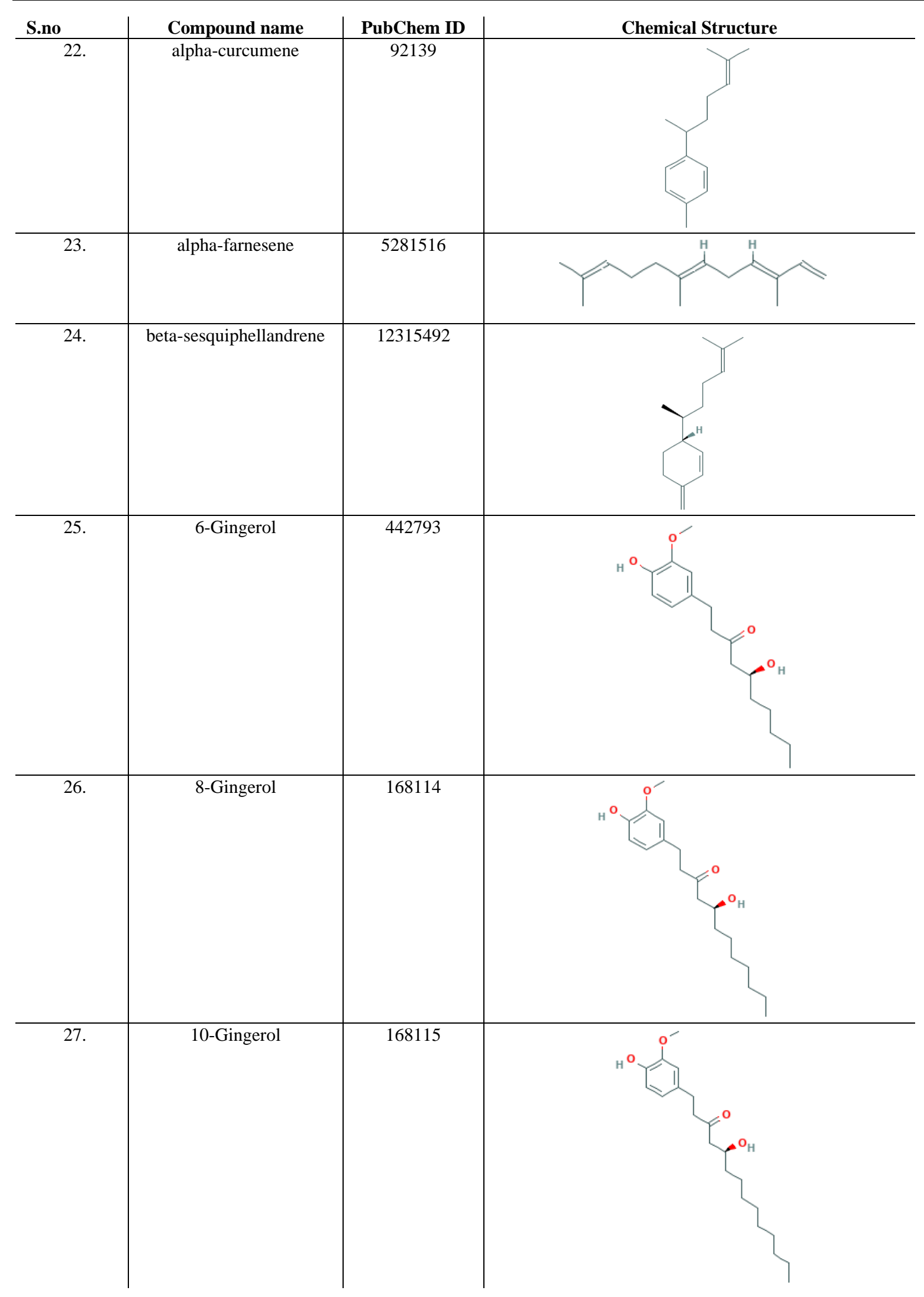

\subsection{Docking procedure.}

Auto dock 4.2 software was utilized to perform target-ligand docking. Scoring of the target-ligand association was done based on free binding energy [22]. The Lamarckian hereditary calculation (LGA) was applied to elucidate the collaboration design between the COVID-19 and the natural compounds of black pepper ginger and clove [23]. In all docking strategies, 10 free hereditary calculations were run. A population size of 150 for every atom 
under investigation LGA pursue was halted the greatest number of 2500000 energy assessments and 27,000 most extreme ages. The auto dock was then executed to acquire Docking Log Files (DLG) for additional examination.

\subsection{ADME analysis property.}

The ADME parameters of the various compounds from Black pepper (Piper nigrum), Clove (Syzygium aromaticum), and Ginger (Zingiber officinale roscoe) that indicated best outcomes were investigated by SwissADME (SwissADME url:- http://www.swissadme.ch) [24].

\section{Results and Discussion}

The medication resemblance properties, for example, an atomic load of the compound (Molecular Weight), number of hydrogen bond donor (HBD) and acceptor (HBA), and determined LogP of Piper nigrum, Syzygium aromaticum, and Zingiber officinale roscoe derived compounds, were initially assessed by Lipinski's standard of five. Table 4, 5, and 6 listed the medication resemblance properties of Piper nigrum, Syzygium aromaticum, and Zingiber officinale roscoe. According to Lipinski's rule of five:

$\checkmark$ Molecular weight of the compound should be less than 500 daltons

$\checkmark$ Hydrogen bond accepter range should be less than 10

$\checkmark$ Hydrogen bond donor range should be less than 5

$\checkmark \quad$ And the value of Log P should be less than 5

\subsection{Selection of screened phytocompounds for docking studies.}

In order to screen a potential lead candidate for COVID-19 treatment, we have selected 26 compounds from Black pepper (Piper nigrum), Clove (Syzygium aromaticum), and Ginger (Zingiber officinale roscoe) for this study. A literature review has supported the significant inhibitory potential of these phytocompounds against various viral diseases such as HIV and Hepatitis, through various ways such as inhibition of reverse-transcriptase, DNA polymerase, and protease, etc. We have considered two standard drug references for our study, including Abacavir and hydroxychloroquine, as they are presently being utilized for COVID-19 treatment [25-26].

Table 2. Chemical and physical properties of various compounds from Black pepper (Piper nigrum), Clove

(Syzygium aromaticum), and Ginger (Zingiber officinale roscoe).

\begin{tabular}{|c|c|c|c|c|c|}
\hline Compound Name & Pub chem id & Molecular wt. & $\begin{array}{c}\text { Hydrogen bond } \\
\text { accepter }\end{array}$ & $\begin{array}{c}\text { Hydrogen bond } \\
\text { donor }\end{array}$ & $\log p$ \\
\hline Piperanine & 5320618 & $287.35 \mathrm{~g} / \mathrm{mol}$ & 3 & 0 & 3.1 \\
\hline Piperylin A & 636537 & $271.31 \mathrm{~g} / \mathrm{mol}$ & 3 & 0 & 3.1 \\
\hline Delta cadinol & 3084311 & $222.37 \mathrm{~g} / \mathrm{mol}$ & 1 & 1 & 3.3 \\
\hline Delta guaiene & 94275 & $204.35 \mathrm{~g} / \mathrm{mol}$ & 0 & 0 & 4.6 \\
\hline$(\mathrm{Z})(\mathrm{E})$ farnesol & 445070 & $222.37 \mathrm{~g} / \mathrm{mol}$ & 1 & 1 & 4.8 \\
\hline (E) beta ocimene & 5281553 & $136.23 \mathrm{~g} / \mathrm{mol}$ & 0 & 0 & 4.3 \\
\hline Guaiol & 227829 & $222.37 \mathrm{~g} / \mathrm{mol}$ & 1 & 1 & 3.1 \\
\hline Eugenol & 3314 & $164.2 \mathrm{~g} / \mathrm{mol}$ & 2 & 1 & 2 \\
\hline Eugenol acetate & 7136 & $206.24 \mathrm{~g} / \mathrm{mol}$ & 3 & 0 & 2.3 \\
\hline Eugenol benzoate & 62362 & $268.31 \mathrm{~g} / \mathrm{mol}$ & 3 & 0 & 4.4 \\
\hline Methyl eugenol & 7127 & $178.23 \mathrm{~g} / \mathrm{mol}$ & 2 & 0 & 2.5 \\
\hline Triterpenoids & 71597391 & $472.7 \mathrm{~g} / \mathrm{mol}$ & 5 & 4 & 3.9 \\
\hline Eugenin & 10189 & $206.19 \mathrm{~g} / \mathrm{mol}$ & 4 & 1 & 1.2 \\
\hline Eugenitin & 3083581 & $220.22 \mathrm{~g} / \mathrm{mol}$ & 4 & 1 & 2.5 \\
\hline Campesterol & 173183 & $400.7 \mathrm{~g} / \mathrm{mol}$ & 1 & 1 & 8.8 \\
\hline \multicolumn{5}{|c|}{ https://biointerfaceresearch.com/ } & 1128 \\
\hline
\end{tabular}




\begin{tabular}{l|c|c|c|c|c} 
Compound Name & Pub chem id & Molecular wt. & $\begin{array}{c}\text { Hydrogen bond } \\
\text { accepter }\end{array}$ & $\begin{array}{c}\text { Hydrogen bond } \\
\text { donor }\end{array}$ & Log p \\
\hline Shogaol & 5281794 & $276.4 \mathrm{~g} / \mathrm{mol}$ & 3 & 1 & 3.7 \\
\hline Paradol & 94378 & $278.4 \mathrm{~g} / \mathrm{mol}$ & 3 & 1 & 3.8 \\
\hline Zingerone & 31211 & $194.23 \mathrm{~g} / \mathrm{mol}$ & 3 & 1 & 0.8 \\
\hline 6-dehydrogingerdione & 22321203 & $290.4 \mathrm{~g} / \mathrm{mol}$ & 4 & 2 & 4.2 \\
\hline Gingeronone A & 5281775 & $356.4 \mathrm{~g} / \mathrm{mol}$ & 5 & 2 & 3.7 \\
\hline beta-bisabolene & 10104370 & $204.35 \mathrm{~g} / \mathrm{mol}$ & 0 & 0 & 5.2 \\
\hline alpha-curcumene & 92139 & $202.33 \mathrm{~g} / \mathrm{mol}$ & 0 & 0 & 5.4 \\
\hline alpha-farnesene & 5281516 & $204.35 \mathrm{~g} / \mathrm{mol}$ & 0 & 0 & 6.1 \\
\hline beta-sesquiphellandrene & 12315492 & $204.35 \mathrm{~g} / \mathrm{mol}$ & 0 & & 5.4 \\
\hline 6-Gingerol & 442793 & $294.4 \mathrm{~g} / \mathrm{mol}$ & 4 & 2 & 2.5 \\
\hline 8-Gingerol & 168114 & $322.4 \mathrm{~g} / \mathrm{mol}$ & 4 & 2 & 4.2 \\
\hline 10-Gingerol & 168115 & $350.5 \mathrm{~g} / \mathrm{mol}$ & 4 & & 2 \\
\hline
\end{tabular}

\subsection{Molecular docking using AutoDock 4.2 software.}

Then we performed molecular docking by using AutoDock tool 4.2. The binding energies and docking results of the derived compounds from black pepper, clove, and ginger against 6LU7 are listed below in tables 7, 8, and 9. Out of 26 selected compounds, Guaiol has shown the best inhibitory potential (maximum binding energy) against the 6LU7 target of COVID-19 (Table 3). Gingeronone A has shown the best inhibitory potential (maximum binding energy) against the 7JTL target of COVID-19 (Table 4) (Figure 2). Additionally, comparative analysis of these two compounds with standard drugs has also depicted better inhibitory potential (maximum binding energy) against these two targets (Table $5 \&$ Table 6) (Figure 3 \& Figure 4). Thus both the screened phytocompounds could be further utilized for in vitro studies to elucidate a potent lead candidate for drug development against COVID-19.

Table 3. Docking results of 6LU7 (COVID-19) with several compounds of black pepper, clove, and ginger.

\begin{tabular}{|c|c|c|c|c|c|c|}
\hline \multirow[b]{2}{*}{ S.no } & \multirow[b]{2}{*}{ Compounds } & \multicolumn{5}{|c|}{ Autodock } \\
\hline & & $\begin{array}{c}\text { Binding } \\
\text { Energy } \\
\text { (kcal/mol) }\end{array}$ & $\begin{array}{c}\text { No. of } \\
\text { hydrogen } \\
\text { bonds }\end{array}$ & $\begin{array}{c}\text { Total } \\
\text { Internal } \\
\text { Energy }\end{array}$ & $\begin{array}{c}\text { Estimated } \\
\text { inhibition } \\
\text { constant }(\mathbf{K i})\end{array}$ & Residues \\
\hline 1. & Piperanine & -4.54 & 1 & -0.41 & 470.97 & Target:A:GLN127:HN \\
\hline 2. & Piperylin A & -4.88 & 2 & -0.17 & 264.24 & $\begin{array}{l}\text { Target:A:GLN107:HN } \\
\text { Target:A:ILE152:O }\end{array}$ \\
\hline 3. & Delta cadinol & -6.42 & 2 & -0.2 & 19.67 & $\begin{array}{l}\text { Target:A:THR111:HN: } \\
\text { Target:A:THR111:OG1 }\end{array}$ \\
\hline 4. & Delta guaiene & -5.73 & $\ldots \ldots$ & 0.17 & 63.59 & \\
\hline 5. & $(Z)(E)$ farnesol & -3.52 & 3 & -0.58 & 2.62 & $\begin{array}{l}\text { Target:A:ALA129:HN: } \\
\text { Target:A:LYS137:HN: } \\
\text { Target:A:ALA129:O }\end{array}$ \\
\hline 6. & (E) beta ocimene & -3.51 & $\ldots \ldots$ & -0.17 & 2.64 & $\ldots \ldots$ \\
\hline 7. & Guaiol & -6.50 & 2 & -0.19 & 17.13 & $\begin{array}{l}\text { Target:A:THR111:HN: } \\
\text { Target:A:THR111:OG1 }\end{array}$ \\
\hline 8. & Eugenol & -4.85 & 3 & -0.27 & 280.63 & $\begin{array}{l}\text { Target:A:THR111:HN: } \\
\text { Target:A:GLN110:HE21 } \\
\text { Target:A:THR111:OG1 }\end{array}$ \\
\hline 9. & Eugenol acetate & -4.66 & 2 & -0.4 & $382 . .51$ & $\begin{array}{l}\text { Target:A;GLN110:HE21 } \\
\text { Target:A:THR111:HN: }\end{array}$ \\
\hline 10. & $\begin{array}{l}\text { Eugenol } \\
\text { benzoate }\end{array}$ & -4.41 & 1 & -0.92 & 587.85 & Target:A:GLN127:O \\
\hline 11. & Methyl eugenol & -4.35 & 1 & -0.38 & 649.74 & Target:A:GLN110:HE21 \\
\hline 12. & Triterpenoids & -5.53 & 2 & -0.37 & 87.89 & $\begin{array}{l}\text { Trget:A:LYS5:HZ3: } \\
\text { Target:A:GLY170:O }\end{array}$ \\
\hline 13. & Eugenin & -4.76 & 2 & -0.55 & 326.61 & $\begin{array}{l}\text { Target:A:GLN110:HE21 } \\
\text { Target:A:THR111:HN: }\end{array}$ \\
\hline 14. & Eugenitin & -5.22 & 1 & -0.59 & 150.18 & Target:A:GLN110HE21: \\
\hline 15. & Campesterol & -4.41 & $\ldots$ & -0.9 & 251.61 & $\ldots$ \\
\hline 16. & Shogaol & -4.12 & 2 & -1.37 & 962.19 & $\begin{array}{c}\text { Target:A:GLU290:OE1 } \\
\text { Target:A:LYS5:HZ2 }\end{array}$ \\
\hline
\end{tabular}




\begin{tabular}{|c|c|c|c|c|c|c|}
\hline \multirow[b]{2}{*}{ S.no } & \multirow[b]{2}{*}{ Compounds } & \multicolumn{5}{|c|}{ Autodock } \\
\hline & & $\begin{array}{c}\text { Binding } \\
\text { Energy } \\
(\mathrm{kcal} / \mathrm{mol})\end{array}$ & $\begin{array}{c}\text { No. of } \\
\text { hydrogen } \\
\text { bonds }\end{array}$ & $\begin{array}{c}\text { Total } \\
\text { Internal } \\
\text { Energy }\end{array}$ & $\begin{array}{c}\text { Estimated } \\
\text { inhibition } \\
\text { constant }(\mathbf{K i})\end{array}$ & Residues \\
\hline 17. & Paradol & -3.43 & $\ldots \ldots$ & -1.22 & 3.06 & $\ldots \ldots \ldots \ldots$ \\
\hline 18. & Zingerone & -5.30 & 4 & -0.34 & 131.04 & $\begin{array}{c}\text { Target:A:THR111:HN: } \\
\text { Target:A:SER158:HG: } \\
\text { Target:A:GLN110:HE21 } \\
\quad: \\
\text { Target:A:THR111:OG1 }\end{array}$ \\
\hline 19. & $\begin{array}{c}6- \\
\text { dehydrogingerdi } \\
\text { one }\end{array}$ & -4.55 & 1 & -0.75 & 461.48 & Target:A:ARG105:O \\
\hline 20. & Gingeronone A & -4.8 & 1 & -1.76 & 305.41 & Target:A:GLY138:O \\
\hline 21. & beta-bisabolene & -4.07 & $\ldots \ldots$ & -0.49 & 1.04 & ..................... \\
\hline 22. & alpha-curcumene & -4.64 & $\ldots \ldots \ldots$ & -0.6 & 394.74 & $\ldots \ldots \ldots \ldots \ldots \ldots$ \\
\hline 23. & alpha-farnesene & -4.1 & f....... & -0.33 & 958.18 & \\
\hline 24. & $\begin{array}{c}\text { beta- } \\
\text { sesquiphellandre } \\
\text { ne }\end{array}$ & -5.43 & $\ldots \ldots$ & -0.73 & 103.95 & (n..... \\
\hline 25. & 6-Gingerol & -3.21 & 1 & -1.38 & 4.46 & Target:A:LYS5:HZ2: \\
\hline 26. & 8-Gingerol & -3.22 & 2 & -1.31 & 4.38 & $\begin{array}{l}\text { Target:A:LYS5:HZ2: } \\
\text { Target:A:GLU290:O }\end{array}$ \\
\hline 27. & 10-Gingerol & -2.53 & 1 & -1.93 & 13.95 & Target:A:LYS137:O \\
\hline
\end{tabular}

Table 4. Docking results of 7JTL (SARS Cov-2) with several compounds of black pepper, clove, and ginger.

\begin{tabular}{|c|c|c|c|c|c|c|}
\hline \multirow[b]{2}{*}{ S.no } & \multirow[b]{2}{*}{ Compounds } & \multicolumn{5}{|c|}{ Autodock } \\
\hline & & $\begin{array}{l}\text { Binding } \\
\text { Energy } \\
\text { (kcal/mol) }\end{array}$ & $\begin{array}{l}\text { No. of } \\
\text { hydrogen } \\
\text { bonds }\end{array}$ & $\begin{array}{l}\text { Total } \\
\text { Internal } \\
\text { Energy }\end{array}$ & $\begin{array}{l}\text { Estimated } \\
\text { inhibition } \\
\text { constant }(\mathbf{K i})\end{array}$ & Residues \\
\hline 1 & Piperanine & -6.66 & 2 & -0.36 & 13.22 & $\begin{array}{l}\text { Target:A:VAL33:O } \\
\text { Target:A:CYS61:HN: }\end{array}$ \\
\hline 2 & Piperylin A & -6.55 & 2 & -0.29 & 14.71 & $\begin{array}{l}\text { Target:A:TRP45:HN: } \\
\text { Target:A:CYS61:HN: }\end{array}$ \\
\hline 3 & Delta cadinol & 5.94 & 1 & -0.2 & 44.55 & Target:A:LEU60:O \\
\hline 4 & Delta guaiene & -5.98 & $\ldots \ldots$ & -018 & 41.45 & \\
\hline 5 & $(Z)(E)$ farnesol & -5.72 & 3 & -0.55 & 64.55 & $\begin{array}{l}\text { Target:A:TRP45:HN: } \\
\text { Target:A:CYS61:HN: } \\
\text { Target:A:TRP45:O }\end{array}$ \\
\hline 6 & (E) beta ocimene & -4.09 & ......... & -0.26 & 1.00 & $\ldots \ldots \ldots \ldots \ldots$ \\
\hline 7 & Guaiol & -6.38 & 1 & -0.18 & 21.21 & Target:A:TYR73:O \\
\hline 8 & Eugenol & -5.33 & 1 & -0.48 & 123.29 & Target:A:CYS61:HN: \\
\hline 9 & Eugenol acetate & -5.08 & 3 & -0.46 & 189.60 & $\begin{array}{l}\text { Target:A:CYS61:HN: } \\
\text { Target:A:TRP45:HN: } \\
\text { Target:A:SER43:O }\end{array}$ \\
\hline 10 & $\begin{array}{l}\text { Eugenol } \\
\text { benzoate }\end{array}$ & -6.19 & 2 & -0.73 & 28.80 & $\begin{array}{l}\text { Target:A:TRP45:HE1: } \\
\text { Target:A:PHE86:HN: }\end{array}$ \\
\hline 11 & Methyl eugenol & -4.82 & 2 & -0.35 & 294.39 & $\begin{array}{l}\text { Target:A:TRP45:HE1: } \\
\text { Target:A:PHE86:HN: }\end{array}$ \\
\hline 12 & Triterpenoids & -6.12 & 2 & -0.41 & 32.92 & $\begin{array}{l}\text { Target:A:VAL33:O } \\
\text { Target:A:PHE86:O }\end{array}$ \\
\hline 13 & Eugenin & -5.97 & 1 & -0.57 & 42.28 & Target:A:CYS61:HN: \\
\hline 14 & Eugenitin & 24.73 & 2 & -0.58 & & $\begin{array}{l}\text { Target:A:LEU60:HN: } \\
\text { Target:A:CYS61:HN: }\end{array}$ \\
\hline 15 & Campesterol & -7.23 & & -0.75 & 5.00 & \\
\hline 16 & Shogaol & -5.96 & 1 & -1.16 & 42.48 & Target:A:PHE86:HN: \\
\hline 17 & Paradol & -5.16 & 1 & -1.08 & 165.48 & Target:A:PHE86:HN: \\
\hline 18 & Zingerone & -5.74 & 2 & -0.56 & 62.84 & $\begin{array}{l}\text { Target:A:CYS61:HN: } \\
\text { Target:A:PHE86:HN: }\end{array}$ \\
\hline 19 & $\begin{array}{l}\text { 6- } \\
\text { dehydrogingerdi } \\
\text { one }\end{array}$ & -5.85 & 1 & -1.26 & 51.75 & Target:A:TRP45:HN: \\
\hline 20 & Gingeronone A & -7.65 & 2 & -1.36 & 2.49 & $\begin{array}{l}\text { Target:A:TRP45:HN: } \\
\text { Target:A:ASP35:OD2 }\end{array}$ \\
\hline 21 & beta-bisabolene & -5.89 & $\ldots \ldots$ & -0.41 & 47.99 & $\ldots \ldots \ldots \ldots \ldots \ldots \ldots$ \\
\hline 22 & $\begin{array}{l}\text { alpha- } \\
\text { curcumene }\end{array}$ & -5.58 & $\ldots \ldots$ & -0.35 & 81.87 & .................. \\
\hline 23 & alpha-farnesene & -5.45 & ....... & -0.39 & 101.72 & \\
\hline
\end{tabular}




\begin{tabular}{|c|c|c|c|c|c|c|}
\hline \multirow[b]{2}{*}{ S.no } & \multirow[b]{2}{*}{ Compounds } & \multicolumn{5}{|c|}{ Autodock } \\
\hline & & $\begin{array}{l}\text { Binding } \\
\text { Energy } \\
\text { (kcal/mol) }\end{array}$ & $\begin{array}{l}\text { No. of } \\
\text { hydrogen } \\
\text { bonds }\end{array}$ & $\begin{array}{l}\text { Total } \\
\text { Internal } \\
\text { Energy }\end{array}$ & $\begin{array}{l}\text { Estimated } \\
\text { inhibition } \\
\text { constant (Ki) }\end{array}$ & Residues \\
\hline 24 & $\begin{array}{l}\text { beta- } \\
\text { sesquiphellandre } \\
\text { ne }\end{array}$ & -6.13 & ….... & -0.58 & 31.98 & ...... \\
\hline 25 & 6-Gingerol & -4.27 & 1 & -1.1 & 736.81 & Target:A:PHE86:HN \\
\hline 26 & 8-Gingerol & -4.7 & 2 & -1.16 & 356.1 & $\begin{array}{l}\text { Target:A:TRP45:HN: } \\
\text { Target:A:SER43:O }\end{array}$ \\
\hline 27 & 10-Gingerol & -5.66 & 1 & -1.18 & 71.05 & Target:A:CYS61:HN: \\
\hline
\end{tabular}

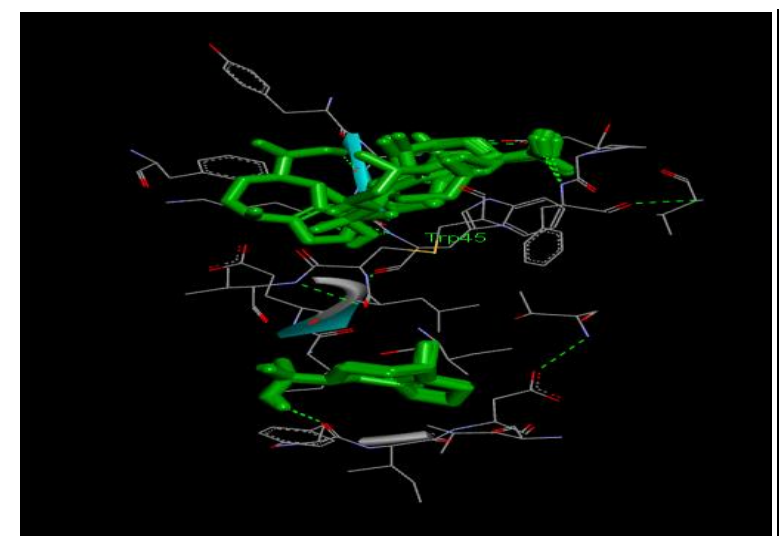

Guaiol

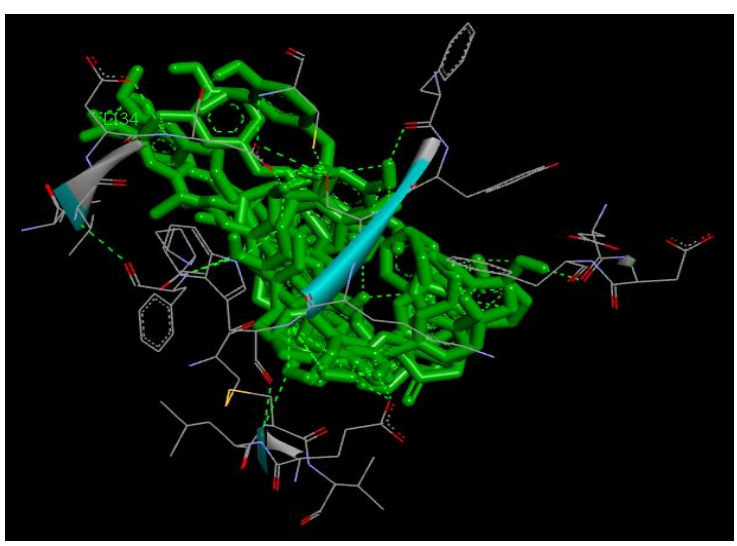

Gingeronone-A

Figure 2. Best screened compound showing maximum binding energy against two selected targets of co (a) 6LU7 and (b)7JTL binding with black pepper, clove, and ginger has shown the best binding energies.

Table 5. Docking results of 6LU7 (COVID-19) with hydroxychloroquine.

\begin{tabular}{l|c|c|c|c|c|c} 
S.No & Compounds & \multicolumn{5}{|c}{ Autodock } \\
\hline & & $\begin{array}{c}\text { Binding Energy } \\
(\mathbf{k c a l} / \mathbf{m o l})\end{array}$ & $\begin{array}{c}\text { No. of } \\
\text { hydrogen } \\
\text { bonds }\end{array}$ & $\begin{array}{c}\text { Total } \\
\text { Internal } \\
\text { Energy }\end{array}$ & $\begin{array}{c}\text { Estimated } \\
\text { inhibition } \\
\text { constant (Ki) }\end{array}$ & Residues \\
\hline 1 & Hydroxychloroquine & -3.81 & $\ldots \ldots \ldots$ & -2.6 & 1.61 & $\ldots \ldots \ldots$ \\
\hline 2 & Remdesivir & -3.52 & 2 & -2.73 & 2.63 & $\begin{array}{c}\text { Target:A:GLY138:O } \\
\text { Target:A:LYS137:O }\end{array}$
\end{tabular}

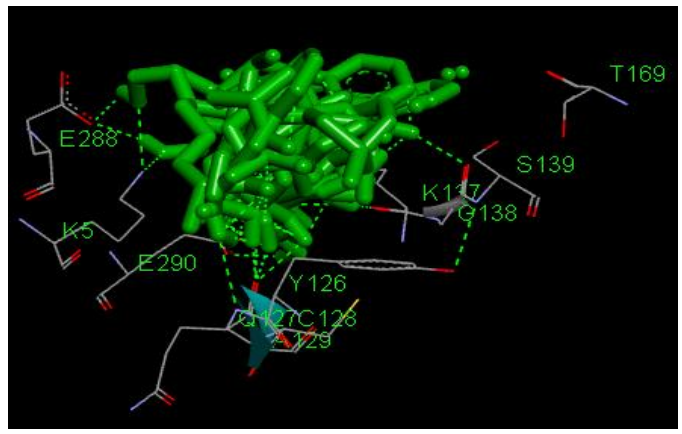

(a)

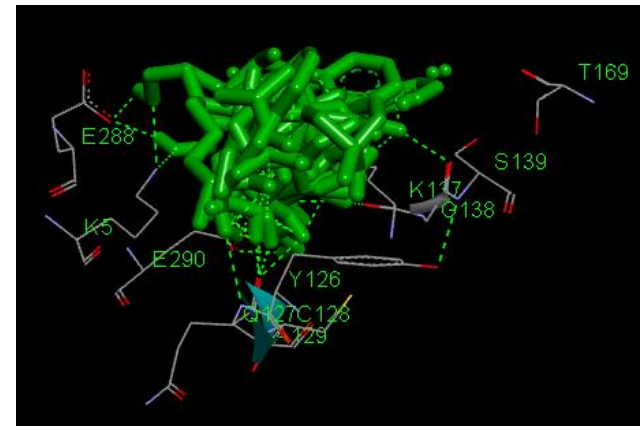

(b)

Figure 3. Docking analysis of 6LU7 with two standard drugs (a) Hydroxychloroquine and (b) Remdesivir.

Table 6. Docking results of 7JTL (SARS Cov-2) with hydroxychloroquine.

\begin{tabular}{|c|c|c|c|c|c|c|}
\hline S.No & Compounds & Autodock & & & & \\
\hline & & $\begin{array}{l}\text { Binding } \\
\text { Energy } \\
\text { (kcal/mol) }\end{array}$ & $\begin{array}{l}\text { No. of } \\
\text { hydrogen } \\
\text { bonds }\end{array}$ & $\begin{array}{l}\text { Total } \\
\text { Internal } \\
\text { Energy }\end{array}$ & $\begin{array}{l}\text { Estimated } \\
\text { inhibition } \\
\text { constant }(\mathrm{Ki})\end{array}$ & Residues \\
\hline 1 & Hydroxychloroquine & -5.56 & 2 & -0.3 & 84.47 & $\begin{array}{l}\text { Target:A:GLU59:OE } \\
2 \\
\text { Target:A:GLU106:O }\end{array}$ \\
\hline 2 & Remdesivir & -3.15 & 1 & 0.01 & 4.94 & Target:A:CYS83:O \\
\hline
\end{tabular}




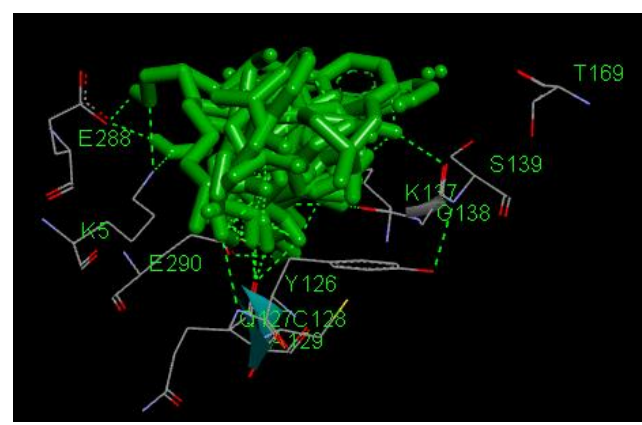

(a)

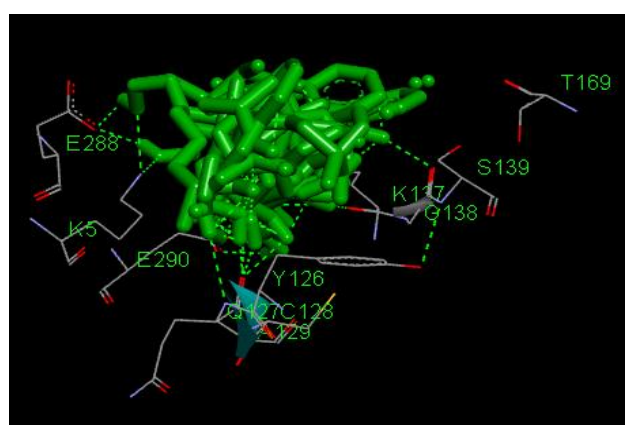

(b)

Figure 4. Docking analysis of 6LU7 with two standard drugs (a) Hydroxychloroquine and (b) Remdesivir.

\section{Conclusions}

Our current investigation endeavored to investigate the capability of successful natural mixes from black pepper, clove, and ginger against the principle protease of COVID-19 in contrast with the proposed drug hydroxychloroquine and remdesivir. We have chosen powerful natural mixes from these black pepper, clove, and ginger against COVID-19 objective protein 6LU7 and 7JTL. Our outcomes from molecular docking strongly suggested that Guaiol and Gingeronone A showed the best restricting viability against COVID-19 Main Proteases, which can urge us to analyze its potential in pre-clinical and clinical examinations.

\section{Funding}

This research received no external funding.

\section{Acknowledgments}

The authors thank Noida Institute of Engineering \& Technology management for providing the facilities to carry out this study.

\section{Conflicts of Interest}

The authors declare no conflict of interest.

\section{References}

1. Zu, Z.Y.; Jiang, M.D.; Xu, P.P.; Chen, W.; Ni, Q.Q.; Lu, G.M.; Zhang, L.J. Coronavirus Disease 2019 (COVID-19): A Perspective from China. Radiology 2020, 296, E15-E25, https://doi.org/10.1148/radiol.2020200490.

2. Abdirizak, F.; Lewis, R.; Chowell, G. Evaluating the potential impact of targeted vaccination strategies against severe acute respiratory syndrome coronavirus (SARS-CoV) and Middle East respiratory syndrome coronavirus (MERS-CoV) outbreaks in the healthcare setting. Theoretical Biology and Medical Modelling 2019, 16, https://doi.org/10.1186/s12976-019-0112-6.

3. Hosseiny, M.; Kooraki, S.; Gholamrezanezhad, A.; Reddy, S.; Myers, L. Radiology Perspective of Coronavirus Disease 2019 (COVID-19): Lessons From Severe Acute Respiratory Syndrome and Middle East Respiratory Syndrome. American Journal of Roentgenology 2020, 214, 1078-1082, https://doi.org/10.2214/AJR.20.22969.

4. Momattin, H.; Mohammed, K.; Zumla, A.; Memish, Z.A.; Al-Tawfiq, J.A. Therapeutic Options for Middle East Respiratory Syndrome Coronavirus (MERS-CoV) \&\#x2013; possible lessons from a systematic review of SARS-CoV therapy. International Journal of Infectious Diseases 2013, 17, e792-e798, https://doi.org/10.1016/j.ijid.2013.07.002.

5. Lee, P.-I.; Hsueh, P.-R. Emerging threats from zoonotic coronaviruses-from SARS and MERS to 2019nCoV. Journal of Microbiology, Immunology and Infection 2020, 53, 365-367, https://doi.org/10.1016/j.jmii.2020.02.001. 
6. Beigel, J.H.; Tomashek, K.M.; Dodd, L.E.; Mehta, A.K.; Zingman, B.S.; Kalil, A.C.; de Castilla, D.L. Remdesivir for the treatment of Covid-19-preliminary report. The New England journal of medicine 2020, 1-14, https://doi.org/10.1056/NEJMoa2007764.

7. Gao, J.; Tian, Z.; Yang, X. Breakthrough: Chloroquine phosphate has shown apparent efficacy in treatment of COVID-19 associated pneumonia in clinical studies. BioScience Trends 2020, 14, 72-73, https://doi.org/10.5582/bst.2020.01047.

8. Cortegiani, A.; Ingoglia, G.; Ippolito, M.; Giarratano, A.; Einav, S. A systematic review on the efficacy and safety of chloroquine for the treatment of COVID-19. Journal of Critical Care 2020, 57, 279-283, https://doi.org/10.1016/j.jcrc.2020.03.005.

9. Wang, T.; Du, Z.; Zhu, F.; Cao, Z.; An, Y.; Gao, Y.; Jiang, B. Comorbidities and multi-organ injuries in the treatment of COVID-19. The Lancet 2020, 395, https://doi.org/10.1016/S0140-6736(20)30558-4.

10. Liu, Y.; Chan, W. K.; Wang, Z.; Hur, J.; Xie, J.; Yu, H.; He, Y. Ontological and bioinformatic analysis of anti-coronavirus drugs and their Implication for drug repurposing against COVID-19. Pre print 2020, https://doi.org/10.20944/preprints202003.0413.v1.

11. Feng, Z.; Chen, M.; Liang, T.; Shen, M.; Chen, H.; Xie, X.-Q. Virus-CKB: an integrated bioinformatics platform and analysis resource for COVID-19 research. Briefings in Bioinformatics 2020, https://doi.org/10.1093/bib/bbaa155.

12. Huang, X.; He, C.; Hua, X.; Kan, A.; Sun, S.; Wang, J.; Li, S. Bioinformatic Analysis of Correlation between Immune Infiltration and COVID-19 in Cancer Patients. International Journal of Biological Sciences 2020, 16, 2464-2476, https://doi.org/10.7150/ijbs.48639.

13. D’Souza, S.P.; Chavannavar, S.V.; Kanchanashri, B.; Niveditha, S.B. Pharmaceutical Perspectives of Spices and Condiments as Alternative Antimicrobial Remedy. Journal of Evidence-Based Complementary \& Alternative Medicine 2017, 22, 1002-1010, https://doi.org/10.1177/2156587217703214.

14. Yashin, A.; Yashin, Y.; Xia, X.; Nemzer, B. Antioxidant Activity of Spices and Their Impact on Human Health: A Review. Antioxidants 2017, 6, https://doi.org/10.3390/antiox6030070.

15. Rasool, A.; Khan, M.-u.-R.; Ali, M.; Anjum, A.; Ahmed, I.; Aslam, A.; Rafique, G.; Masood, S.; Nawaz, M. Anti-Avian influenza virus H9N2 activity of aqueous extracts of Zingiber officinalis (Ginger) \& Allium sativum (Garlic) in chick embryos. Pakistan Journal of Pharmaceutical Sciences 2017, 30, 1341-1344.

16. Kaushik, S.; Jangra, G.; Kundu, V.; Yadav, J.P.; Kaushik, S. Antiviral activity of Zingiber officinale (Ginger) ingredients against the Chikungunya virus. VirusDisease 2020, 31, 270-276, https://doi.org/10.1007/s13337-020-00584-0.

17. Beristain-Bauza, S.D.C.; Hernández-Carranza, P.; Cid-Pérez, T.S.; Ávila-Sosa, R.; Ruiz-López, I.I.; OchoaVelasco, C.E. Antimicrobial Activity of Ginger (Zingiber Officinale) and Its Application in Food Products. Food Reviews International 2019, 35, 407-426, https://doi.org/10.1080/87559129.2019.1573829.

18. Nag, A.; Chowdhury, R.R. Piperine, an alkaloid of black pepper seeds can effectively inhibit the antiviral enzymes of Dengue and Ebola viruses, an in silico molecular docking study. VirusDisease 2020, 31, 308315, https://doi.org/10.1007/s13337-020-00619-6.

19. Tice, C.M. Selecting the right compounds for screening: does Lipinski's Rule of 5 for pharmaceuticals apply to agrochemicals? Pest Management Science 2001, 57, 3-16, https://doi.org/10.1002/15264998(200101)57:1<3::AID-PS269>3.0.CO;2-6.

20. Athar Abbasi, M.; Raza, H.; Aziz ur, R.; Zahra Siddiqui, S.; Adnan Ali Shah, S.; Hassan, M.; Seo, S.-Y. Synthesis of novel N-(1,3-thiazol-2-yl)benzamide clubbed oxadiazole scaffolds: Urease inhibition, Lipinski rule and molecular docking analyses. Bioorganic Chemistry 2019, 83, 63-75, https://doi.org/10.1016/j.bioorg.2018.10.018.

21. Khan, M.F.; Verma, G.; Akhtar, W.; Shaquiquzzaman, M.; Akhter, M.; Rizvi, M.A.; Alam, M.M. Pharmacophore modeling, 3D-QSAR, docking study and ADME prediction of acyl 1,3,4-thiadiazole amides and sulfonamides as antitubulin agents. Arabian Journal of Chemistry 2019, 12, 5000-5018, https://doi.org/10.1016/j.arabjc.2016.11.004.

22. Castro-Alvarez, A.; Costa, A.M.; Vilarrasa, J. The Performance of Several Docking Programs at Reproducing Protein-Macrolide-Like Crystal Structures. Molecules 2017, 22, https://doi.org/10.3390/molecules22010136.

23. Guo, S.; Chen, Y.; Shi, S.; Wang, X.; Zhang, H.; Zhan, Y.; An, H. Arctigenin, a novel TMEM16A inhibitor for lung adenocarcinoma therapy. Pharmacological Research 2020, 155, https://doi.org/10.1016/j.phrs.2020.104721.

24. Tsaioun, K.; Blaauboer, B.J.; Hartung, T. Evidence-based absorption, distribution, metabolism, excretion (ADME) and its interplay with alternative toxicity methods. Altex 2016, 33, 343358,https://dx.doi.org/10.14573/altex.1610101.

25. Beigel, J.H.; Tomashek, K.M.; Dodd, L.E.; Mehta, A.K.; Zingman, B.S.; Kalil, A.C.; Hohmann, E.; Chu, H.Y.; Luetkemeyer, A.; Kline, S.; Lopez de Castilla, D.; Finberg, R.W.; Dierberg, K.; Tapson, V.; Hsieh, L.; Patterson, T.F.; Paredes, R.; Sweeney, D.A.; Short, W.R.; Touloumi, G.; Lye, D.C.; Ohmagari, N.; Oh, M.-d.; Ruiz-Palacios, G.M.; Benfield, T.; Fätkenheuer, G.; Kortepeter, M.G.; Atmar, R.L.; Creech, C.B.; Lundgren, J.; Babiker, A.G.; Pett, S.; Neaton, J.D.; Burgess, T.H.; Bonnett, T.; Green, M.; Makowski, M.; 
Osinusi, A.; Nayak, S.; Lane, H.C. Remdesivir for the Treatment of Covid-19 — Final Report. New England Journal of Medicine 2020, 383, 1813-1826, https://doi.org/10.1056/NEJMoa2007764.

26. Pandey, P.; Khan, F.; Rana, A.K.; Srivastava, Y.; Jha, S.K.; Jha, N.K. A Drug Repurposing Approach Towards Elucidating the Potential of Flavonoids as COVID-19 Spike Protein Inhibitors. Biointerface Research in Applied Chemistry 2020, 11, 8482-8501, https://dx.doi.org/10.33263/BRIAC111.84828501

27. Pandey, P.; Khan, F.; Kumar, A.; Srivastava, A.; Jha, N.K. Screening of Potent Inhibitors Against 2019 Novel Coronavirus (Covid-19) from Alliumsativum and Allium cepa: An In Silico Approach. Biointerface Research in Applied Chemistry 2020, 11, 7981-7993, https://dx.doi.org/10.33263/BRIAC111.79817993. 